\title{
Simulação matemática dos sinais sonoros do violão através da convolução
}

\author{
Mathematical simulation of the sound signals of the guitar through \\ convolution
}

\author{
1 Paulo Sérgio Teixeira paulost39@gmail.com \\ 2 Alexandre Furtado Ferreira \\ 3 José Flávio Silveira Feiteira
}

\footnotetext{
1 Engenheiro mecânico cursando mestrado em engenharia metalúrgica e materiais. Professor nas áreas de máquinas hidráulicas e sistemas térmicos, mecânica técnica, metrologia, produção mecânica e tecnologia mecânica. Universidade Federal Fluminense - UFF.

2 Professor da Escola de Engenharia Industrial Metalúrgica de Volta Redonda, Universidade Federal Fluminense- PPGEM. Universidade Federal Fluminense, Escola de Engenharia Industrial Metalúrgica de Volta Redonda.

3 Professor da Escola de Engenharia Industrial Metalúrgica de Volta Redonda, Universidade Federal Fluminense - UFF.
}

\section{Resumo}

O som ou sinal sonoro pode ser representado por uma soma de diversas ondas individuais ou pela superposição de sinais mais simples, denominada componentes de Fourier, nas quais cada onda corresponde a uma determinada frequência múltipla da componente inicial. Cada tipo de instrumento musical tem uma espécie de assinatura, um conjunto de características sonoras associado a ele tais como amplitude (ou intensidade) e a frequência de cada modo de vibração perturbado. Embora possa parecer subjetivas, ela tem uma descrição matemática extremamente precisa que torna cada tipo de instrumento único. A convolução é uma forma matemática de combinar dois sinais para formar um terceiro sinal. Trata-se de uma importante técnica do processamento digital de sinais. Usando a estratégia da decomposição pelo impulso, os sistemas são descritos por um sinal denominado de resposta impulsiva. No presente trabalho, o sinal de entrada é representado pela vibração da corda gravada separadamente do violão, a resposta impulsiva do sistema (caixa acústica) é gravada utilizando-se o software Sonelastic e o sinal de saída (convolução) é utilizada a linguagem Matlab.

Palavras-chave:

Sinal sonoro. Fourier. Convolução. Violão. Resposta impulsiva.

\begin{abstract}
The sound or sound signal can be represented by a sum of several individual waves or by the superposition of simpler signals, called Fourier components, in which each wave corresponds to a certain frequency multiple of the initial component. Each type of musical instrument has a kind of signature, a set of sound characteristics associated with it such as amplitude (or intensity) and the frequency of each disturbed vibration mode. While it may seem subjective, it has an extremely accurate mathematical description that makes each type of instrument unique. Convolution is a mathematical way of combining two signals to form a third signal. It is an important technique in digital signal processing. Using the impulse decomposition strategy, the systems are described by a sign called an impulse response. In the present work, the input signal is represented by the vibration of the string recorded separately from the guitar, the impulsive response of the system (speaker box) is recorded using the Sonelastic software and the output signal (convolution) is used the Matlab language.
\end{abstract}

\section{Keywords:}

Sound signal. Fourier. Convolution. Guitar. Impulsive response.

Como você deve citar?

TEIXEIRA, Paulo Sérgio; FERREIRA, Alexandre Furtado; FEITEIRA, José Flávio Silveira. Simulação matemática dos sinais sonoros do violão através da convolução. Cadernos UniFOA, Volta Redonda (RJ), v. 16, n. 46, p. 1-13, ago, 2021. 


\section{INTRODUÇÃO}

\subsection{Sinal Sonoro}

O sinal sonoro resulta da vibração de um meio mecânico ou de uma perturbação mecânica que se propaga através de ondas de pressão no ar que são um caso particular de ondas longitudinais (WUENSCHE, 2009).

Para Lazzarini (1998), o som é uma qualidade perceptiva, resultado da percepção de distúrbios das moléculas de um meio em um certo espaço de tempo. Esses distúrbios, por sua vez, apresentam-se em forma de ondas em sua propagação pelo meio.

Segundo Lacerda (1996), o som pode ser representado por uma soma de diversas ondas individuais, denominada componentes de Fourier, e cada onda corresponde a uma determinada frequência múltipla da componente inicial.

Quando um instrumento musical produz uma determinada nota, ele produz não apenas o som da frequência fundamental, que dá a qualidade de tonalidade, mas também o som de frequências harmônicas múltiplas da fundamental. Se não fosse assim, não haveria diferença entre o som de instrumentos diferentes. É a proporção dos harmônicos e sua intensidade relativa produzidas por cada tipo ou qualidade de instrumento que os diferencia, dando-lhes sua qualidade de timbre. É o que comumente se define como a "cor" do som (FILHO, 2009; DONOSO et al., 2008)

Timbre é a propriedade do som que nos permite reconhecer sua origem. 0 timbre diferencia, "personaliza" o som. Por meio do timbre, identificamos "o que" ou qual instrumento está produzindo o som (RAMALHO JÚNIOR et al., 1999).

Cada tipo de instrumento musical tem uma espécie de assinatura, um conjunto de características sonoras associado a ele. Embora possam parecer subjetivas, elas têm uma descrição matemática extremamente precisa que torna cada tipo de instrumento único. A mesma nota emitida por um violão soa diferente, quando produzida por um piano. Isso acontece porque, embora a frequência fundamental seja a mesma em ambos os instrumentos, o som de instrumentos musicais não é simples. As amplitudes e o tempo de duração de cada um dos harmônicos presentes no som resultante podem variar independentemente. A combinação dessas duas propriedades tem o nome de timbre (DODGE, 1997).

A mesma nota tocada em instrumentos diferentes soará com timbres diferentes pelo fato de 0 som gerado por cada instrumento conter os harmônicos do tom fundamental em proporções diferentes. Essas proporções são características de cada tipo de instrumento, e, para o ouvido mais acurado, características de cada modelo ou exemplar de instrumento (ROGERS, 2013; HALLIDAY et al., 2009).

O sinal sonoro pode ser representado como uma superposição de sinais mais simples, reproduzido por uma série de Fourier onde a intensidade sonora de cada frequência harmônica componente é determinada pelas características do instrumento. Por exemplo, o som produzido por instrumentos de corda geralmente possui todos os múltiplos da frequência fundamental como componentes, enquanto alguns instrumentos de sopro, que possuem tubo de ressonância aberto em um extremo e fechado em outro, produzem som que contém apenas os harmônicos ímpares. Dessa forma, o som produzido por um clarinete (figura 1) contém quase que somente harmônicos ímpares; já no som de uma flauta ou oboé, o primeiro e o segundo harmônico estão presentes (praticamente só eles) (SNIEDER,1994; HALLIDAY et al., 2009). 
Figura 1 - Representação de alguns instrumentos musicais no domínio tempo (esquerda) e no domínio da frequência (direita).

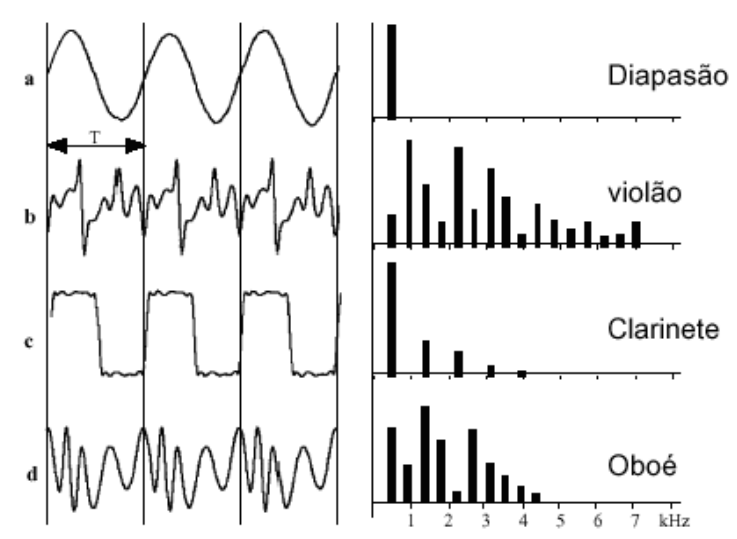

Fonte: Wuensche (2009)

\subsection{Análise Sonora de Instrumentos Musicais}

Para analisar os sinais acústicos de instrumentos, nesse caso, o violão, é necessário considerá-lo com um sistema vibrante. Os sistemas de vibração podem vibrar em vários modos diferentes. Cada modo é relacionado a uma frequência diferente e, portanto, um modo de vibração pode ser excitado individualmente por algum tipo de perturbação relacionado à certa frequência (SANTOS, 2013).

A descrição desse movimento vibracional é bastante difícil, pois é necessário saber a amplitude (ou intensidade), a frequência de cada modo de vibração perturbado e a taxa de amortecimento no meio material em que se propaga.

O espectro de uma vibração indica quais frequências estão sendo excitadas e com que intensidade. Essa análise espectral, também conhecida como análise no domínio da frequência, é obtida através da análise de Fourier do sinal sonoro, especificamente pela aplicação da transformada de Fourier ao sinal temporal representativo da vibração estudada (TEIXEIRA et al., 2014).

Após a teoria de Fourier, que se propunha decompor funções periódicas em somas de funções senoidais, surgiu a transformada de Fourier e depois a transformada rápida de Fourier (FFT - Fast Fourier Transform).

Uma função pode ser convertida do domínio do tempo para o da frequência através de um operador matemático chamado genericamente de transformada integral. A Transformada de Fourier decompõe uma função na soma de um (potencialmente infinito) número de componentes senoidais, produzindo um espectro de frequências. A transformada inversa correspondente converte esse espectro de volta para o domínio do tempo, ou seja, para a função original (CARILLO, 2014).

Ao aplicar-se a transformada de Fourier, passa-se do domínio do tempo para o domínio da frequência e a informação a respeito de deslocamentos de fase do sinal em função da frequência desaparece.

Em acústica e música, a fundamental é a mais baixa frequência e a de maior amplitude das componentes da série harmônica de um som. Tecnicamente, a fundamental corresponde ao primeiro harmônico. Na realidade, o som é composto de várias frequências, mas as que se destacam são as que entram em ressonância, fenômeno que acontece quando a vibração atinge a chamada frequência natural 
de vibração do meio. A frequência fundamental ou a primeira que entra em ressonância é responsável pela percepção da altura de uma nota, enquanto que as demais, chamadas de harmônicos, participam da composição da forma de onda do som (TEIXEIRA, 2015).

\subsection{Sinais no Domínio do Tempo}

Domínio do tempo é um termo utilizado para descrever a análise de funções matemáticas com relação ao tempo. Nesse domínio, o valor da função é conhecido a cada instante para o caso do tempo contínuo (figura 2 a) ou em vários instantes separados, no caso do tempo discreto (figura 2 b).

Figura 2 - (a) Sinal contínuo; (b) Sinal discreto

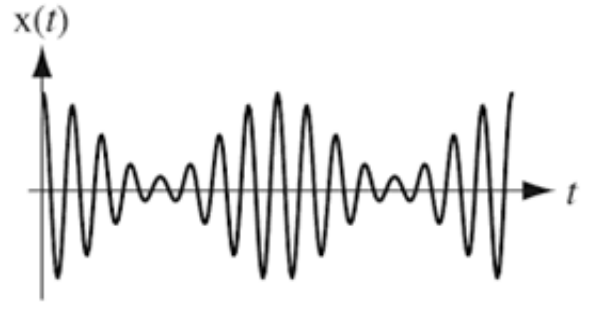

(a)

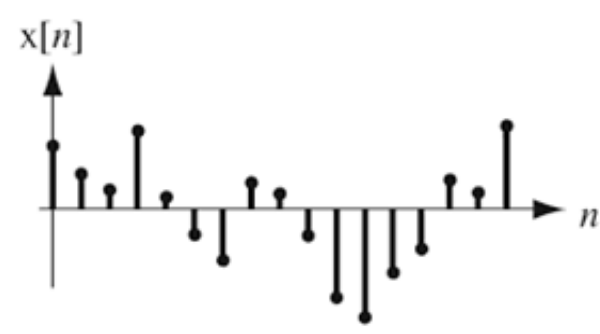

(b)

Fonte: Pinto (2018)

A representação no domínio do tempo indica de que forma a amplitude do sinal varia com o tempo e, em certos casos, fornece algumas indicações acerca da variação do conteúdo em frequência do sinal. 0 espectro de um sinal no domínio do tempo é representado por uma onda que pode variar a sua amplitude no tempo. Em instrumentos musicais, o formato dessas ondas para uma mesma nota musical pode variar bastante de acordo com o tipo de instrumento, pois possuem timbres diferentes (figura 3 a).

\subsection{Sinais no Domínio da Frequência}

O domínio da frequência designa a representação de funções matemáticas com relação à frequência, em contraste com a análise no domínio do tempo. 0 domínio da frequência pressupõe periodicidade no tempo (eq. 1), isto é, para existir um mapeamento entre os domínios do tempo e da frequência deve-se assumir que os fenômenos no domínio do tempo se repetem em intervalos iguais a T.

$$
f=\frac{1}{T}
$$

Tudo se passa como se o domínio da frequência enxergasse o domínio do tempo sob a óptica de intervalos regulares de tempo. Para se perceber melhor as vantagens que essa representação de sinais pode trazer, toma-se um sinal com representação simples nos dois domínios: a senoide.

Na representação do domínio da frequência (figura 3 b), o mesmo sinal é representado apenas pelos seus parâmetros, ficando subentendida a função temporal escolhida com referência na decomposição: 
Figura 3 - (a) Formas de ondas de diferentes instrumentos para uma mesma nota musical.

(b) Sinal no domínio da frequência.

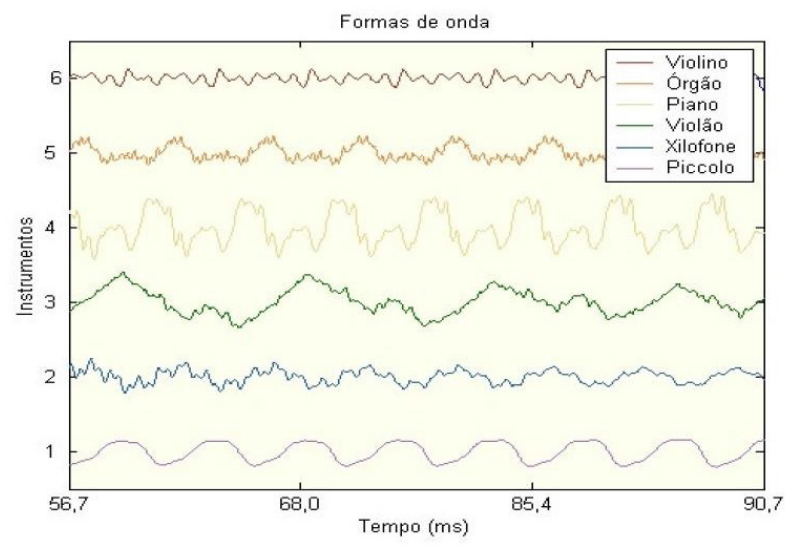

(a)

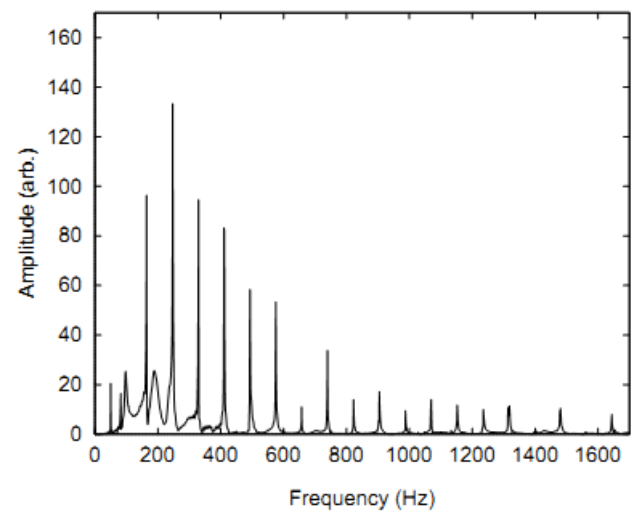

(b)

Fonte: Pinto (2018)

Fonte: Wright (1996)

Esse processo pode ser mais bem entendido através da Figura 4, a qual ilustra a transformada de Fourier de um sinal. Desse modo, é possível visualizá-lo no domínio do tempo e sua respectiva transformada no domínio da frequência. Assim, pode-se afirmar que a Transformada de Fourier decompõe um sinal em suas componentes elementares seno e cosseno (DA PENHA, 1999).

Figura 4 - Sinal no Domínio do Tempo e sua Respectiva Transformada de Fourier (Frequência)

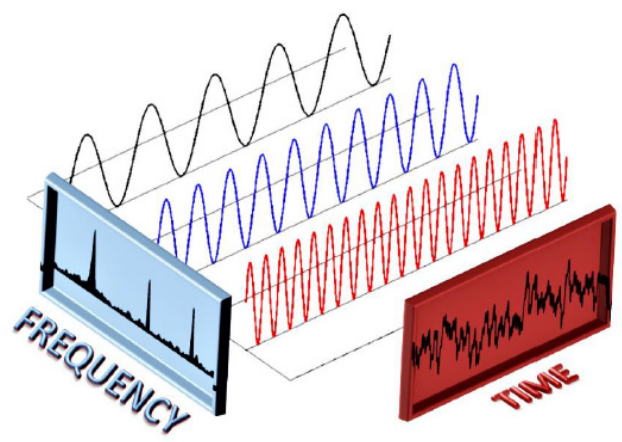

Fonte: Pinto (2018)

\subsection{Convolução}

Convolução é uma operação realizada em dois sinais que envolvem a multiplicação de um sinal por uma versão atrasada ou deslocada de outro sinal, integrando ou calculando a média do produto e repetindo o processo para diferentes atrasos. É um processo útil porque descreve com precisão alguns efeitos que ocorrem amplamente em medições científicas. Um exemplo é a influência de um filtro de frequência em um sinal elétrico ou da passagem de banda espectral de um espectrômetro na forma de um espectro óptico registrado, que causa o sinal a ser espalhado no tempo e reduzido na amplitude do pico (O'HAVER, 2008).

Segundo Katznelson (1976), convolução é um operador linear que, a partir de duas funções dadas, resulta em uma terceira que mede a área subentendida pela superposição das mesmas em função do deslocamento existente entre elas. 
O teorema da convolução estabelece que a transformada de Fourier de uma convolução de duas funções é igual ao produto ponto a ponto das transformadas de Fourier de cada função. Em outras palavras, convolução em um domínio (no domínio do tempo) equivale à multiplicação ponto a ponto no outro domínio (no domínio da frequência).

Dadas as funções $f$ e $g$ cujo produto $h(x)=f(x) g(x)$, a convolução entre $f$ e $g$, denotada por $f_{*} g$ (eq. 2), é definida por qualquer uma das integrais:

$$
\left(f_{*} g\right)(x)=\int_{-\infty}^{+\infty} f(x-\omega) g(\omega) d \omega=\int_{-\infty}^{+\infty} f(\omega) g(x-\omega) d \omega
$$

\subsection{Convolução e Resposta Impulsiva}

A convolução é uma forma matemática de combinar dois sinais para formar um terceiro sinal, sendo uma importante técnica do processamento digital de sinais. Usando a estratégia da decomposição pelo impulso, os sistemas são descritos por um sinal denominado de resposta impulsiva. A convolução relaciona os três sinais de interesse: o sinal de entrada, o sinal de saída e a resposta impulsiva do sistema.

Como o interesse deste trabalho é analisar as características de um instrumento de corda, a resposta impulsiva representa, nesse caso, todas as frequências possíveis da caixa acústica do violão. Não se pode dizer que a corda é que vai gerar um impulso capaz de excitar todas as frequências naturais do violão, mas que, notadamente, ela irradia a vibração imposta pela corda à caixa e, possivelmente, há uma resposta a essa vibração.

O objetivo do presente trabalho é estudar sobre as características relevantes à resposta dada por um instrumento de corda a partir de respostas sonoras gravadas e analisadas nos domínios de tempo e frequência através do estudo da convolução de sinais, estabelecendo a relação entre a excitação da corda e a resposta dada pela caixa acústica do violão na formação do som.

\section{MATERIAIS E MÉTODOS}

Para a caracterização dos sinais do instrumento de corda, foram gravadas respostas sonoras de um violão.

As respostas sonoras foram produzidas a partir de dedilhamento de notas musicais com base na escala musical para instrumentos de afinação temperada. Cada nota foi tocada separadamente, abafando-se as demais para não haver interferência de vibração (figura 5 a).

O violão escolhido foi da marca Gianini, sete cordas, tampo de cedro e corda Gianini ginga. Foi criado também um dispositivo de madeira com as mesmas dimensões do braço do violão, medido da cabeça até o cavalete (figura 5 b). Ele serve para se analisar os sinais da corda sem a resposta da caixa. 
Figura 5 - (a) Cordas abafadas com espuma; (b) Dispositivo para Simular braço do Violão; (c) Afinação da corda no dispositivo; (d) Violão pendurado no suporte; (e) Excitação por impulso no cavalete.

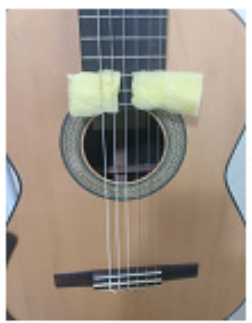

(a)

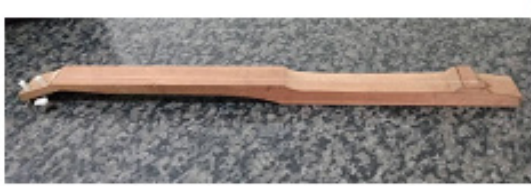

(b)

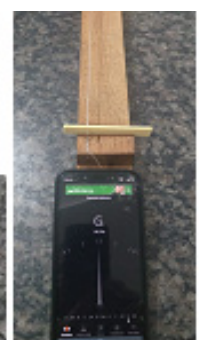

(c)

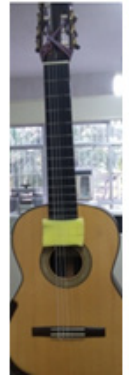

(d)

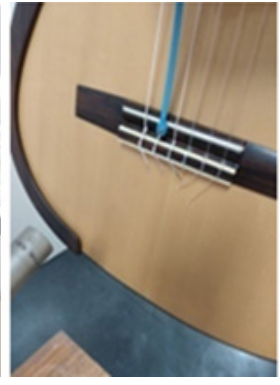

(e)

Fonte: próprios autores (2020)

Para a captação das respostas sonoras do violão, inicialmente, foi aplicada a tensão característica da corda, conhecida como afinação (figura $5 \mathrm{c}$ ). E, em seguida, foi usado um microfone para a gravação dos sinais, utilizando-se o software Cool Edit 2000. Além disso, outros sinais, denominados respostas impulsivas, foram gravados em um software de nome Sonelastic, (TEIXEIRA et al., 2021) que se utiliza de instrumentos auxiliares para reprodução e captação de respostas impulsivas de diversos materiais. Nesse caso, ele foi utilizado para reproduzir a resposta impulsiva do tampo do violão, mais precisamente, no cavalete. 0 presente trabalho utiliza-se da técnica de excitação por impulso através deste software (OTANI, 2017), que consiste, nesse caso, em promover um leve impacto na ponte do violão ao lado da posição de cada corda do instrumento, captando as respostas em frequências naturais através de microfone (Figura 5 e).

Para isso, o instrumento foi pendurado pela cabeça por cordas elásticas em um suporte (Figura 5 d). Nas cordas do vilão, foi inserida a tensão de afinação e, em seguida, abafadas com auxílio de espuma, para que não houvesse interferência de vibração das cordas como proposto por Cossolino e Pereira (2010).

Todos os sinais mencionados foram gravados em arquivos digitais de áudio com extensão "wav".

A primeira etapa depois da gravação é a transferência dos sinais para serem lidos e processados no software Matlab R2010, gerando-se gráficos de amplitude versus tempo. Em seguida, é feita a conversão para o domínio da frequência através da transformada rápida de Fourier (FFT) (TEIXEIRA, 2014).

Como a convolução representa a operação que, a partir de duas funções dadas, resulta em uma terceira, no caso do violão, é representada cada nota como sendo a convolução do sinal gerado pela vibração da corda com o sinal gerado pela vibração do instrumento e mais significativamente pelo tampo, ou seja, a resposta ao impulso gerado pela própria corda. Os sinais sonoros das cordas foram gravados com auxílio do instrumento que simula o braço do violão tocando a corda a quinze centímetros do cavalete, semelhante à posição que é tocada no instrumento. A resposta da caixa ou, mais precisamente, do tampo foi gravada no software Sonelastic, que tem como acessório um pequeno instrumento capaz de promover um leve impulso. 0 software, por sua vez, grava a resposta a esse impulso. Essa resposta contém várias frequências naturais, que, possivelmente, seriam excitadas através da corda vibrando. E, ainda, foi gravado no violão, para efeito de comparação, um sinal com a mesma corda utilizada no instrumento que simula o braço.

Para essa gravação, se fez necessário que a corda estivesse posicionada com a mesma tensão de afinação, para que o tampo tenha o mesmo comportamento de quando o instrumento está sendo 
tocado. Foi tocada uma corda por vez e as demais foram abafadas, para se evitar que elas vibrassem e isso pudesse interferir no sinal que estava sendo gerado.

\subsection{Convolução de Sinais}

Nas rotinas do quadro 1, desenvolvidas em linguagem Matlab, é demonstrado um exemplo de aplicação, em que o sinal resposta impulsiva de um violão Gianini adquirido através do software Sonelastic é convoluído com outro sinal gerado pela gravação do áudio, no software Cool edit 2000, da corda Lá, tocada no instrumento que simula o braço do violão. $O$ resultado é um terceiro sinal que representa a convolução. A corda utilizada no dispositivo foi a corda Lá ou $\mathrm{A}_{2}$ com frequência fundamental de $110 \mathrm{Hertz}$. E, por fim, é feita comparação do sinal convoluído com o sinal gravado do mesmo instrumento com a mesma corda e afinação. 
Quadro 1 - Rotina para Convolução de Sinais.

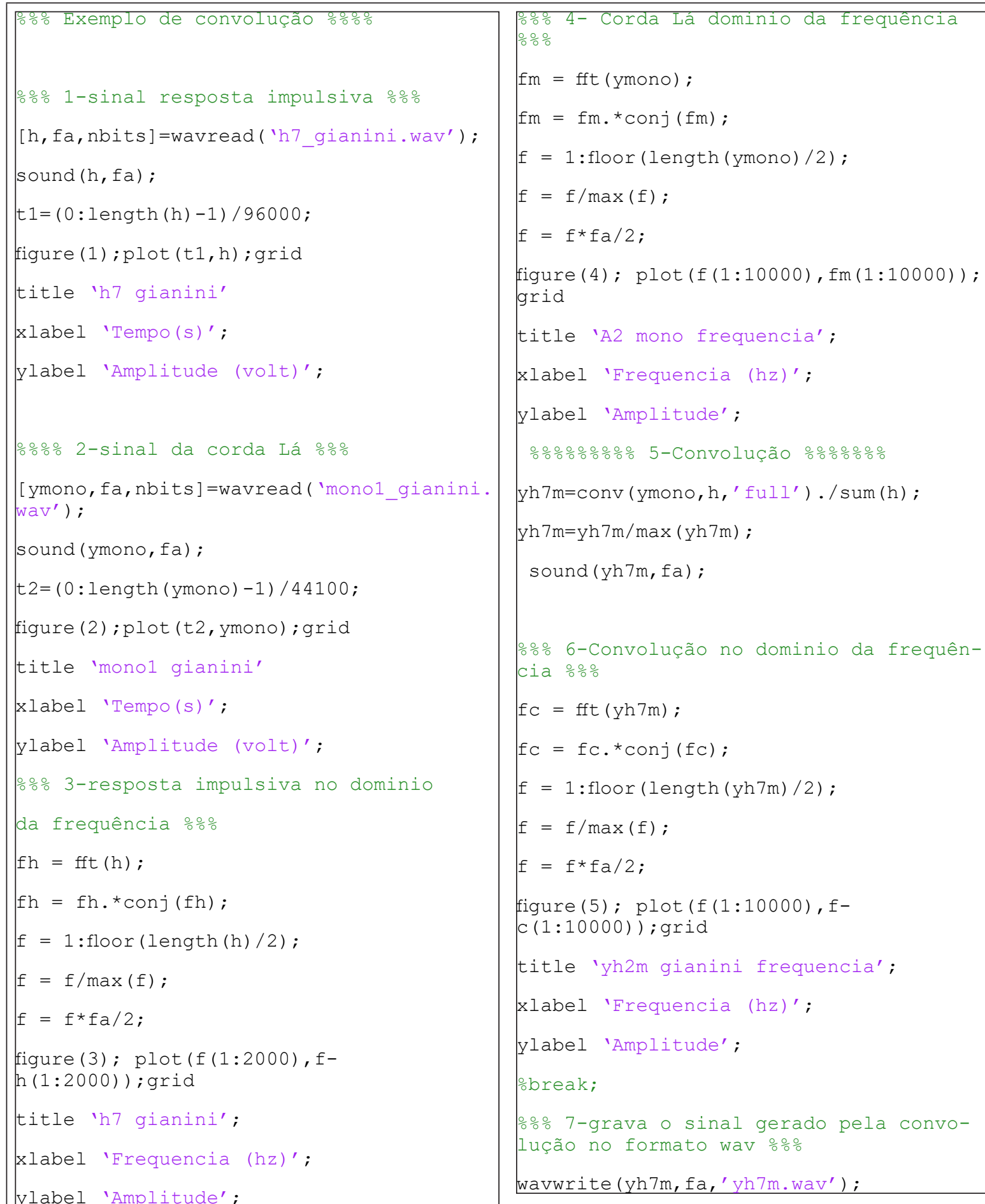




\section{RESULTADOS E DISCUSSÕES}

Nas figuras 8, 9, 10 e 11 estão demonstrados os sinais sonoros processados digitalmente que geram o sinal convoluído, fazendo-se a comparação com o sinal gravado do violão.

Figura 8 - (a) Resposta impulsiva no domínio do tempo; (b) Resposta impulsiva no domínio da frequência

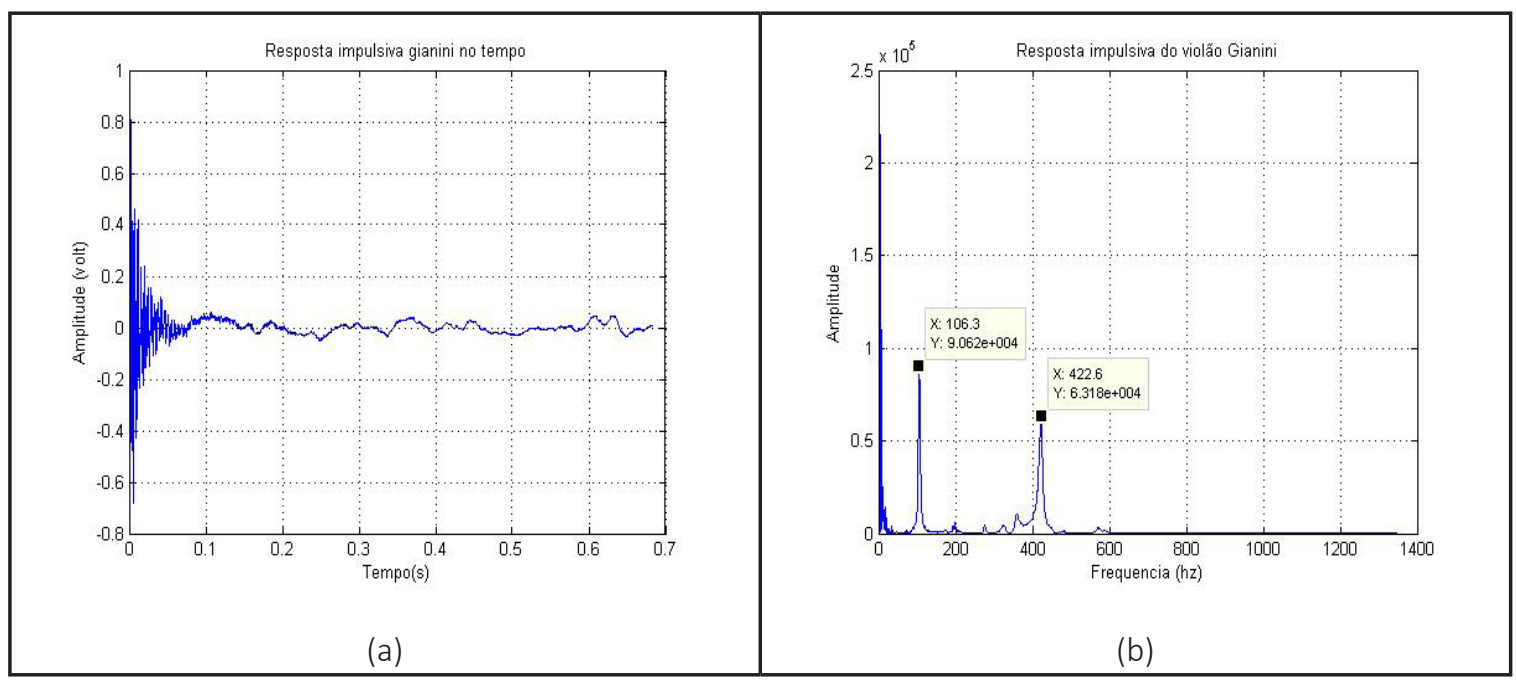

Fonte: próprios autores (2020)

Figura 9 - (a) Corda Lá sem caixa no domínio do tempo;

(b) Corda Lá sem caixa no domínio da frequência

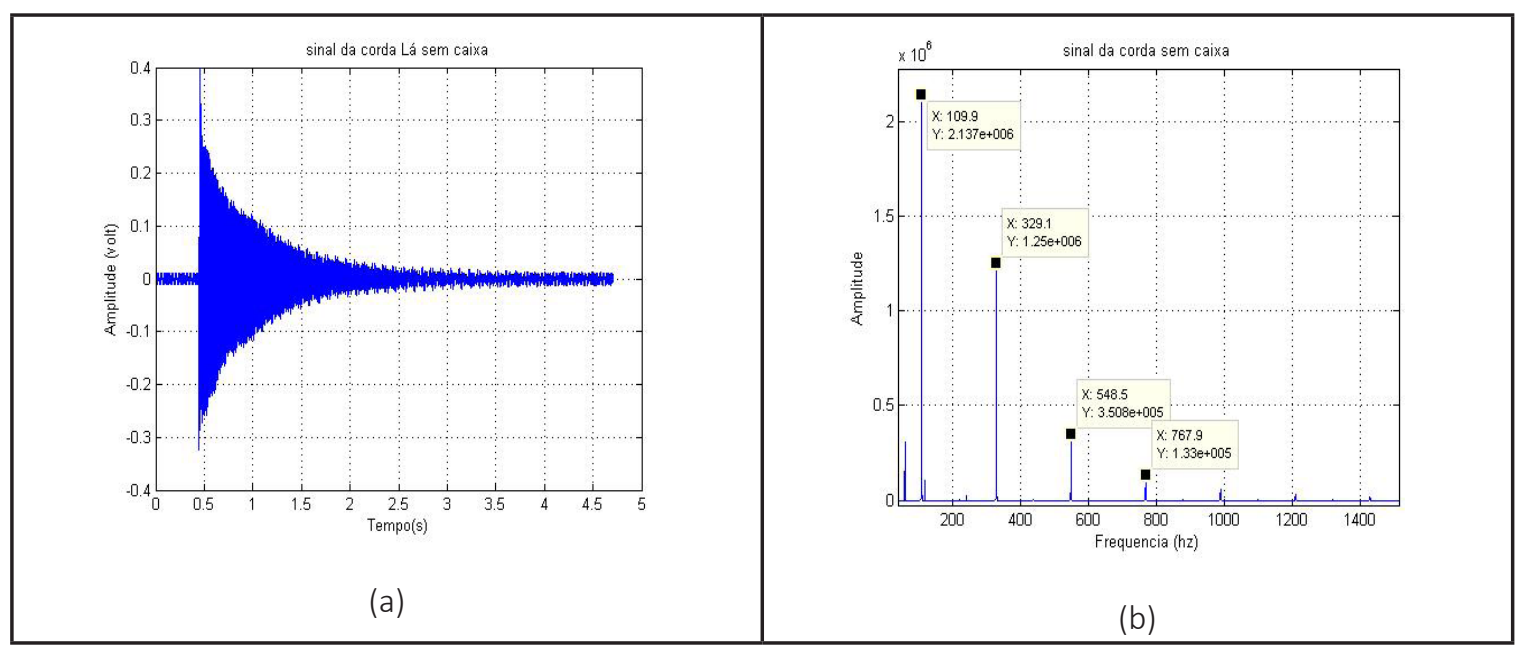

Fonte: próprios autores (2020). 
Figura 10 - (a) Convolução no domínio do tempo; (b) Convolução no domínio da frequência

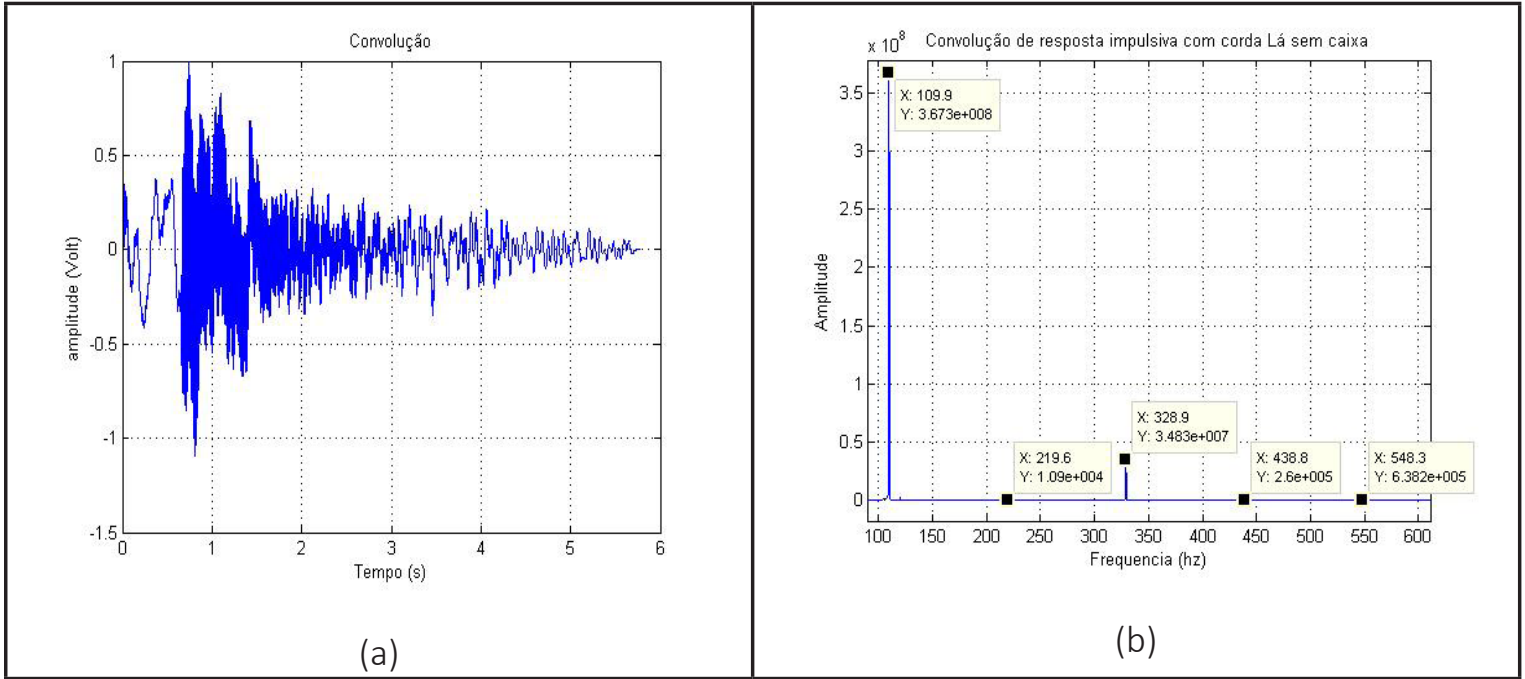

Fonte: próprios autores (2020)

Figura 11 - (a) Sinal medido no violão no domínio do tempo;

(b) Sinal medido no violão no domínio da frequência

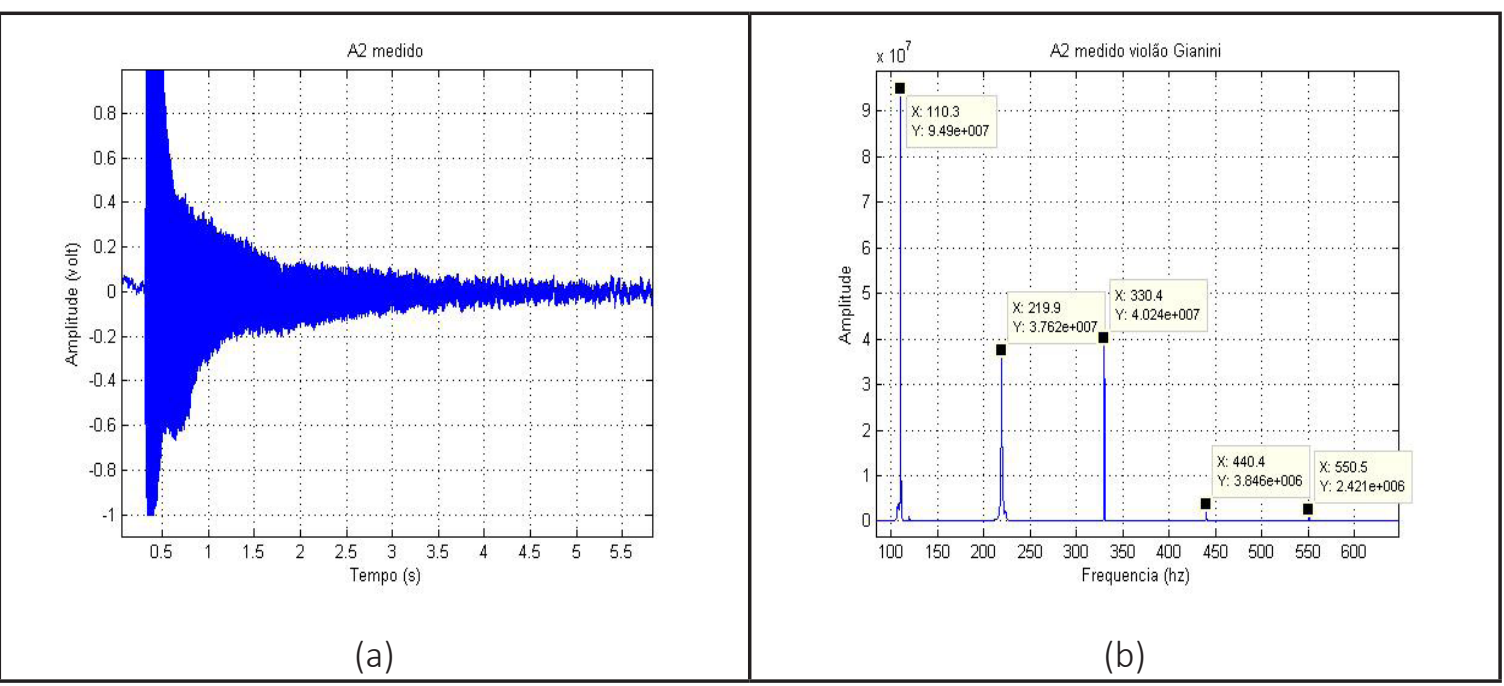

Fonte: próprios autores (2020)

As figuras 8 (a) e (b) representam o sinal resposta impulsiva no domínio do tempo e frequência, respectivamente, que é convoluído com o sinal da corda Lá $\left(A_{2}\right)$ representada também nos domínio do tempo e frequência na figura 9 (a) e (b).

O resultado da convolução está representado na figura 10 (a) e (b), nos domínios do tempo e frequência, nos quais aparece com amplitudes mais evidentes a frequência fundamental com 110,3 Hertz e, o segundo harmônico, com 328,9 Hertz. Os demais harmônicos apresentam amplitudes discretas com relação a estes.

Na comparação dos sinais, convoluído (figura 10 a e b) e medido (figura 11 a e b), no violão Gianini, o resultado da convolução mostra-se bastante satisfatório com grandes amplitudes para a frequência fundamental, segundo e terceiro harmônico nos dois sinais, porém há diferenças com relação às amplitudes. 
Os resultados dessa comparação poderiam ter valores de amplitudes mais próximos, mas é difícil calibrar a mesma energia gerada na resposta que a caixa do violão emprega ao se tocar a corda do violão e a energia do impulso gerada no Sonelastic.

\section{CONCLUSÃO}

$\mathrm{Na}$ análise da convolução de sinais, conclui-se que os sinais convoluídos e medidos apresentam características semelhantes de frequências (fundamental e harmônicos), com algumas diferenças em relação às amplitudes. A frequência fundamental apresenta amplitude elevada, maior até que o sinal medido, suprimindo graficamente as amplitudes das demais frequências. Com relação à resposta ao impulso gerado sinteticamente pelo aparelho (Sonelastic), não se pode dizer que represente fielmente à resposta da caixa do violão à vibração da corda. $E$, isso se deve, muito provavelmente, pela energia imposta pela excitação por impulso não ser com a mesma intensidade ou que essa vibração se propague no meio de maneira diferente da provocada pela corda ou que também seja pelo fato de que o aparelho gere um sinal de, aproximadamente, 0,7 segundos e as notas usadas tenham, no mínimo, 3 segundos. Entretanto, por outro lado, ao se ouvir os sinais sonoros, convoluído e medido, percebe-se certa semelhança entre eles.

\section{REFERÊNCIAS}

CARILLO, S. Fourier Series. ResearchGate, 2014. DOI: 10.1007/978-94-007-2739-7_23

COSSOLINO L. C.; PEREIRA A. H. A. Amortecimento: classificação e métodos de determinação (Informativo Técnico Científico). Universidade de São Carlos, 2010.

DA PENHA, R. M. L. Análise de Sinais em Regime Transiente Aplicando a Técnica de Wavelet. 1999. Tese de Doutorado. Universidade de São Paulo,1999.

DODGE, C.; JERSE, T. A. Computer music synthesis, composition, and performance. 2 ed. New York: Charmer Books.1997.

DONOSO, J.P.; TANNÚS, A.; GUIMARÃES, F. e FREITAS, T.C. A física do Violino. Revista Brasileira do Ensino de Física 30, 2. 2008.

FILHO, Jorge Costa Pires - Classificação de Instrumentos Musicais em Configurações monofônicas e Polifônicas. Dissertação (Mestrado em Engenharia Elétrica). COPPE, Universidade Federal do Rio de Janeiro, 2009.

HALLIDAY, D.; RESNICK, R. e WALKER, J. Fundamentos de Física 2: Gravitação, Ondas e Termodinâmica. 8. ed. Rio de Janeiro: Editora LTC. 2009.

KATZNELSON, Y. An introduction to Harmonic Analysis. 2. ed. Dover, 1976.

LACERDA, O. - Compêndio de teoria elementar da música. 9. ed. São Paulo: Editora Ricordi Brasileira S.A. 1996.

LAZZARINI, V. E. P. Elementos de Acústica. Music Department. National University of Ireland, Maynooth, 1998. 
O'HAVER, T. A Pragmatic Introduction to Signal Processing. Department of Chemistry and Biochemistry, University of Maryland at College Park, 2008. Disponível em: https://terpconnect.umd.edu/ toh/spectrum/ Acesso em: 30 maio 2020.

OTANI, L.B.; SEGUNDINHO, P.G.A.; MORALES, E. A. M.; PEREIRA, A.H.A. Caracterização dos módulos elásticos de madeiras e derivados utilizando a Técnica de Excitação por Impulso (ITC-05 /ATCP). ATCP Engenharia Física, 2017.

PINTO, A. A. Processamento de Sinais Sonoros para Identificação de Notas Musicais Utilizando o Raspberry Pi. CEFET-MG, Divinópolis, 2018.

RAMALHO JÚNIOR, F.; FERRARO, N.; TOLEDO, P. Fundamentos da física: Termologia, Óptica e Ondas. Vol. 2 São Paulo: Editora Moderna. 1999.

ROGERS, w. F. Physics of Music: Science and Art. Westmont College, Santa Barbara, 2013.

SANTOS, E. M.; MOLINA, C.; TUFAILE, A. P. B. Violão e guitarra como ferramentas para o ensino de física. v. 35, n. 2, 2507. Revista Brasileira de Ensino de Física, 2013.

SNIEDER, R. A Guided Tour of Mathematical Physics. Samizdat Press. Utrecht, 1994.

TEIXEIRA, P. S. Análise e Síntese de Sinais de Instrumentos Dedilhados. Em exemplo: O Violão. Dissertação (Mestrado em Engenharia Metalúrgica e Materiais). Universidade Federal Fluminense. Brasil, 2015.

TEIXEIRA, P. S.; FERREIRA, A. F.; FEITEIRA, J. F. Avaliação das respostas em frequências naturais de um violão pelo método de excitação por impulso e de convolução de sinais. Research, Society and Development, v. 10, n. 2, e24410212491, 2021 | ISSN 2525-3409 | DOI: http://dx.doi.org/10.33448/ rsd-v10i2.12491

TEIXEIRA, P. S.; SILVA, A. J.; FEITEIRA, J. F. Avaliação e comparação de características de amortecimento de sinais gerados de diferentes violões. Cadernos UniFOA, 2014.

WRIGHT, H. The Acoustics and Psichoacoustics of the Guitar. (Ph.D.Thesis )Department of Physics and Astronomy. University of Wales. 1996.

WUENSCHE, Carlos A. Física da Música. Inpe/mct. Divisão de Astrofísica, 2009. Disponível em: http:// www.das.inpe.br/ alex/FisicadaMusica/fismus_introducao.htm. Acesso em: 15 jun. 2020. 